\title{
INFRARED EMISSION-LINES AND THE STELLAR
}

\section{TEMPERATURE}

\author{
SUELI M. VIEGAS and RUTH GRUENWALD \\ Instituto Astronômico e Geofísico - USP, Av. Miguel Stefano, 4200, 04301-002 São \\ Paulo, SP Brazil
}

Observations of near infrared emission-lines are becoming available and can be a powerful tool to improve our knowledge on planetary nebulae properties. For wavelengths in the range 1 to $5 \mu \mathrm{m}$, the emission-lines correspond to atomic transitions of high ionized species of heavy elements. In particular, the [Si VI] $1.96 \mu \mathrm{m}$ and [Si VII] $2.48 \mu \mathrm{m}$ lines have already been detected (Ashley and Hyland, 1988).

The presence of such ions indicates a high stellar temperature $\left(T_{*}\right)$, and intensity line ratios can provide a good stellar temperature indicator for objects for which other methods do not give reliable $T_{*}$ estimations. For example, the Zanstra method gives only a lower limit for stellar temperatures higher than $150000 \mathrm{~K}$; this method is also affected by the nebula optical depth. The Stoy method also fails at high stellar temperatures (Stasinska and Tylenda, 1986).

We analyze here the dependence of the line intensity ratio [Si VI] $1.96 \mu \mathrm{m} /[\mathrm{Si}$ VII] $2.48 \mu \mathrm{m}$ with stellar temperature using the photoionization code Aangaba (Gruenwald and Viegas, 1992) and typical PN conditions $\left(n_{H}=10^{2}-10^{6} \mathrm{~cm}^{-3} ; T_{*}=150000-\right.$ $400000 \mathrm{~K} ; L_{*}=1000-10000 L_{\odot} ;[Z]=$ Stasinska and Tylenda, 1986). The above line intensity ratio decreases with the gas density $\left(7 \%\right.$ from $n_{H}=10^{2}$ to $10^{4} \mathrm{~cm}^{-3} ; 47 \%$ from $n_{H}=10^{2}$ to $10^{6} \mathrm{~cm}^{-3}$ ) but depends very little on the stellar luminosity. This line intensity ratio is a good indicator of stellar temperatures between 150000 and $350000 \mathrm{~K}$.

Presently, there are only two PNe for which intensities of [SiVI] and [SiVII] infrared lines were measured. We apply the proposed method for stellar temperature determination for these two nebulae, considering stellar luminosities and gas densities given in the literature. Unfortunately, these two nebulae provide only extreme examples. For NGC 6302, only a lower limit for the stellar temperature can be obtained, since the observed intensity ratio points to the flat part of the curve intensity ratio vs. stellar temperature, indicating $T_{*} \geq 350000 \mathrm{~K}$. This is consistent with values obtained with Stoy cases II and III (Pottasch, 1984). For NGC 6537, the observations provide only an upper limit for the [SiVII] line intensity. Therefore, only a lower limit for the line ratio and an upper limit for the stellar temperature $(250000 \mathrm{~K})$ can be obtained. This result is also consistent with previous determinations with the Zanstra method (Pottasch, 1984). Furthermore, the observed intensity of these infrared lines relative to $\mathrm{H} \beta$ can be reproduced with a $\mathrm{Si}$ abundance close to half solar, indicating that in these objects Si is not very depleted.

\section{References}

Ashley, M.C.B. and Hyland, A.R. (1988), Ap.J. 331, 532

Gruenwald, R.B. and Viegas, S.M. (1992), Ap.J.Suppl.Ser. 78, 153

Pottasch, S. (1984), "Planetary Nebulae"

Stasinska, G. and Tylenda, R. (1986) A.\&A. 155,137 